\title{
Editorial
}

Respiration

\section{Out of Breath, Asleep at the Wheel}

\author{
E. Paul Cherniack
}

Miami Veterans Affairs Hospital and the University of Miami Miller School of Medicine, Miami, Fla., USA

Traffic accidents are the most common cause of traumatic death and the tenth most common cause of death in the world [1]. Fifty million people are injured, and 1.2 million die in crashes [1]. Given the enormity of the impact, researchers have attempted to determine which comorbid medical conditions might influence crash rate, and whether restricting drivers from driving with these conditions or treating these conditions will save lives.

The number of traffic deaths caused by sleep apnea worldwide is unknown, but, according to US National Safety Council data for 2000, there were over 800,000 drivers in obstructive sleep apnea syndrome (OSAS)-related accidents, resulting in 1,400 deaths. While this represents only a small fraction of the 41,821 fatalities that occurred in the US that year, it has been estimated that treating all OSAS patients in the US alone would nevertheless save 980 lives and USD 3.18 billion per year [2].

Crashes have often been used as the primary endpoint of driving impairment. However, crashes are rare events in the lives of most drivers, and other forms of unsafe driving behavior can occur prior to an accident, which potentially might be worthy of measurement. The use of questionnaires, driving simulators, and road testing has helped to identify hazardous driving [3].

Attempts have also been made to determine which drivers with OSAS are at the most risk. Sleep questionnaires and polysomnography, and driving simulators, have been used to identify potential risk factors in patients with OSAS [3]. While an association has been noted between symptoms of sleep disorder and poor driving performance, these are independent of the severity of apnea. The sensitivity and specificity of symptoms in identifying crash-prone drivers has not yet been established. Subjects with elevated apnea-hypopnea indices have been noted to be more likely to crash, but studies use different definitions for 'elevated' [4]. The technique of driving simulation has not been perfected to the extent that we can predict who will crash. Whether the uses of screening instruments and procedures can be practically implemented to determine which OSAS patients are most accident-prone remains to be clarified.

The effect of treating OSAS patients on accidents has also been studied. Using a driving simulator, improvements in driving performance have been noted within 2 weeks after instituting continuous positive airway pressure (CPAP) [5]. Reductions in accidents have been previously observed in drivers with OSAS using CPAP, but these have been based on uncontrolled, retrospective studies based on patients' self-reports of accidents [6].

In the current issue of Respiration [6], 80 drivers with OSAS and 80 subjects without OSAS who were referred for investigation of sleep disorders agreed to allow researchers to collect their collision data from their insurance companies 2 years prior to the start of the study, and

\section{KARGER}

Fax +41613061234

E-Mail karger@karger.ch

www.karger.com (c) 2007 S. Karger AG, Basel

0025-7931/07/0741-0015\$23.50/0

Accessible online at:

www.karger.com/res
Dr. E. Paul Cherniack

Miami Veterans Affair Hospital

Miami, FL 33125 (USA)

Tel. +1 305575 7101, Fax +1 3055757218

E-Mail evan.cherniack@va.gov 
to continue for 2 years after its onset. OSAS subjects were treated with CPAP. At the end of the trial, the rate of crashes had decreased by slightly more than half in both groups.

While this study was not designed to determine if CPAP specifically lowers the accident risk, the reduction observed is still good news. As the authors correctly point out, the effect of inclusion into the study might have induced drivers in both groups to become more aware of their driving behavior and led to fewer crashes. Thus the study may indirectly suggest that physicians reminding patients of the patients' own primary role in traffic accident prevention might be an effective intervention.

References

1 Worley H: Population Reference Bureau, 2006. www.prb.org.

2 Sassani A, Findley LJ, Kryger M: Reducing motor-vehicle collisions, costs, and fatalities by treating obstructive sleep apnea syndrome. Sleep 2004;27:453-458.

3 Frey JG: OSAS and driving capacity. Rev Med Suisse 2005;1:1561-1562, 1564.

4 Pichel F, Zamarron C, Magan F, et al: Sustained attention measurements in obstructive sleep apnea and risk of traffic accidents. Respir Med 2006;100:1020-1027.
5 Orth M, Duchna HW, Leidag M: Driving simulator and neuropsychological testing in OSAS before and under CPAP therapy. Eur Respir J 2005;26:898-903.

6 Barbé F, Sunyer J, de la Peña A, et al: Effect of continuous positive airway pressure on the risk of road accidents in sleep apnea patients. Respiration 2007;74:44-49. 